\section{Scintigraphic TNM Staging of Tumors: A Proposition}

TO THE EDITOR: Very often, local nodal metastases and distant metastases in lungs and bones from differentiated thyroid cancer are detected only by ${ }^{131} \mathrm{I}$ whole-body scintigraphy, or more accurately by ${ }^{131} \mathrm{I}$ SPECT/CT, during the first radioablation after total thyroidectomy. In a recently published article, Schmidt et al. (1) showed ${ }^{131}$ I SPECT/CT to have a definite advantage in the demonstration of nodal metastases in the neck from differentiated thyroid cancer. Another recent article, by Spanu et al. (2), reported that ${ }^{131}$ I SPECT/CT had incremental utility for the detection of local nodal and distant metastases in lungs and bones from differentiated thyroid cancer. Chest radiography and even CT can miss early pulmonary and skeletal metastases. Contrast-enhanced $\mathrm{CT}$ is not recommended before radioiodine ablation.

The usefulness of TNM staging as a major prognostic indicator in the management of differentiated thyroid cancer has been reemphasized in recent years (3). Postoperative histopathologic examination of thyroidectomy specimens facilitates pathologic TNM (pTNM) staging but does not take into account other prognostic variables such as distant metastases when they are not detected clinically or by radiologic studies, before thyroidectomy. In this context, the utility of radioiodine scintigraphy is significant.

The following case report illustrates my point well. A 36-y-old man presenting with a $2-\mathrm{cm}$ solitary nodule in the right lobe of the thyroid and enlarged ipsilateral cervical nodes underwent total thyroidectomy and right modified radical neck dissection. His chest radiograph showed no evidence of pulmonary metastases. Histopathologic examination found papillary carcinoma with metastatic cervical nodes, and hence the stage was defined as pT1N1bMx. Preablation whole-body scintigraphy revealed residual thyroid tissue, nodal metastases, and focal as well as diffuse radioiodine accumulation in the lungs bilaterally. Lung uptake was confirmed on SPECT/CT, thus altering the stage of the disease from Mx to M1. Scintigraphy-assisted staging ("sTNM" staging, with the stage in this patient defined as "sT1N1bM1") is more accurate than pTNM staging in this setting. Similarly, with the advent of PET/CT, which is currently done for several oncologic indications, the scintigraphic staging of a tumor can be more precise than the conventional TNM staging.

\section{REFERENCES}

1. Schmidt D, Szikszai A, Linke R, Bautz W, Kuwert T. Impact of ${ }^{131}$ I SPECT/spiral $\mathrm{CT}$ on nodal staging of differentiated thyroid carcinoma at the first radioablation. J Nucl Med. 2009;50:18-23.

2. Spanu A, Solinas ME, Chessa F, Sanna D, Nuvoli S, Madeddu G. ${ }^{131}$ I SPECT/CT in the follow-up of differentiated thyroid carcinoma: incremental value versus planar imaging. J Nucl Med. 2009;50:184-190.

3. Cooper DS, Doherty GM, Haugen BR, et al. Management guidelines for patients with thyroid nodules and differentiated thyroid cancer: The American Thyroid Association Guidelines Taskforce. Thyroid. 2006;16:109-142.

Regi Oommen

Christian Medical College

Vellore, India

DOI: 10.2967/jnumed.109.062745
REPLY: We read with interest the letter by Regi Oommen about potential changes in postoperative pathologic TNM (pTNM) staging brought about by integrating ${ }^{131}$ I whole-body scintigraphy (WBS) with ${ }^{131}$ I SPECT/CT in patients with differentiated thyroid cancer (DTC). In particular, the author has illustrated a DTC case in which preablative ${ }^{131} \mathrm{I}$ WBS and ${ }^{131}$ I SPECT/CT changed the pTNM stage from T1N1bMx to T1N1bM1 when scintigraphy showed distant lung metastases that had been missed on chest radiography. SPECT/CT confirmed the lung uptake.

Among the patients enrolled in our study (1), 9 underwent preablative ${ }^{131} \mathrm{I}$ WBS and ${ }^{131} \mathrm{I}$ SPECT/CT. SPECT/CT was concordant with WBS in 7 of 9 patients, confirming residual tissues seen on planar scanning. In the remaining 2 patients, SPECT/CT was more accurate than WBS: in one patient, SPECT/ CT identified a higher number of residues; in the other, SPECT/ CT detected mediastinal lymph nodes and lung metastases occult on planar scanning, as well as a higher number of bone metastases (some of which were small and in the ribs). Moreover, SPECT/CT was more accurate than WBS in several of our patients studied with diagnostic radioiodine during long-term follow-up after radioiodine ablation, identifying occult locoregional or distant lesions not evident on planar scanning and contributing to selection of the most appropriate radioiodine therapeutic dose or to a change in therapeutic approach in some cases.

Schmidt et al (2) demonstrated that SPECT/CT determines lymph node involvement at the radioablation study more accurately than does planar imaging, altering management in roughly one quarter of DTC patients by upstaging or downstaging their disease.

On the basis of the above results, we conclude, in agreement with Dr. Oommen, that in patients scheduled for preablation diagnostic ${ }^{131}$ I scanning, integration of WBS with SPECT/CT is of extreme importance because of the resulting increase in specificity and sensitivity. In particular, this new procedure has the potential to modify pTNM stage by identifying locoregional iodine-avid lymph node metastases that need to be treated surgically before radioiodine ablation, as well as by detecting distant metastases and thus guiding one to the most appropriate radioiodine therapeutic dose or to a treatment alternative to radioiodine.

A reassessment of the role of ${ }^{131}$ I-WBS in the management of DTC patients is probably necessary in light of the high performance obtainable when ${ }^{131} \mathrm{I}$-WBS is integrated with SPECT/CT.

\section{REFERENCES}

1. Spanu A, Solinas ME, Chessa F, Sanna D, Nuvoli S, Madeddu G. ${ }^{131}$ I SPECT/CT in the follow-up of differentiated thyroid carcinoma: incremental value versus planar imaging. J Nucl Med. 2009;50:184-190.

2. Schmidt D, Szikszai A, Linke R, Bautz W, Kuwert T. Impact of ${ }^{131}$ I SPECT/spiral $\mathrm{CT}$ on nodal staging of differentiated thyroid carcinoma at the first radioablation. J Nucl Med. 2009;50:18-23.

Angela Spanu Giuseppe Madeddu University of Sassari Sassari, Italy 